\title{
Problemáticas sociais e suas relações com a pesquisa e a extensão: contribuições do Programa de Pós-Graduação em Psicologia da UFC
}

João Paulo Pereira Barros. Universidade Federal do Ceará Cássio Adriano Braz de Aquino. Universidade Federal do Ceará

Luciana Lobo Miranda. Universidade Federal do Ceará

Ricardo Pimentel Mello. Universidade Federal do Ceará

Verônica Morais Ximenes. Universidade Federal do Ceará

Veriana de Fátima Rodrigues Colaço. Universidade Federal do Ceará

Aluísio Ferreira de Lima. Universidade Federal do Ceará

Zulmira Aurea Cruz Bomfim. Universidade Federal do Ceará

Walberto Silva dos Santos. Universidade Federal do Ceará

Karla Patrícia Holanda Martins. Universidade Federal do Ceará

\section{Resumo}

Este artigo tem o objetivo de apresentar o Programa de Pós-Graduação em Psicologia da Universidade Federal do Ceará (PPGP-UFC), abordando suas especificidades, seus aspectos históricos, suas linhas de pesquisa e as temáticas nelas trabalhadas. Além disso, são destacados os projetos e as ações de intercâmbios nacionais e internacionais, os impactos sociais e sua relevância socioacadêmica, bem como os desafios que se apresentam na atualidade. O programa procura articular a prática de pesquisa com os desdobramentos advindos da extensão universitária, já que prioriza em sua política de produção de conhecimento a tematização de fenômenos sociais que se apresentam especialmente no contexto nordestino. Ressalta-se, ainda, como tal especificidade tem contribuído tanto para a consolidação de laboratórios vinculados às linhas de pesquisa do programa, quanto para a sua inserção social, com vistas a contribuir para o desenvolvimento local e regional. Por fim, são indicados os principais desafios enfrentados pelo programa no contexto atual.

Palavras-chave: psicologia; pesquisa; extensão; pós-graduação.

\begin{abstract}
Social Problems and its Relationship with the Research and Extension: Contributions of the Graduate Program in Psychology of the UFC. This article aims to present the Post Graduate Program in Psychology of the Federal University of Ceará (PPGP-UFC), addressing its specificities, its historical aspects, its lines of research and the themes worked on them. In addition, projects and actions for national and international exchanges, social impacts and their socio-academic relevance, as well as current challenges are highlighted. The Program seeks to articulate the practice of research with developments arising from university extension, as it prioritizes in its policy of knowledge production the thematization of social phenomena, especially in the context of the Brazilian Northeast. It is noteworthy also how such specificity has contributed both to the consolidation of laboratories linked to the research lines of the PPGP-UFC, as well as to their social inclusion in order to contribute to local and regional development. Finally, the main challenges faced by the Program in the current context are indicated.
\end{abstract}

Keywords: psychology; search; extension; postgraduate studies.

\section{Resumen}

Problemáticas sociales y sus relaciones con la Investigación y la Extensión: Contribuciones del Programa de Postgrado en Psicología de la UFC. Este artículo pretende presentar el Programa de Posgrado en Psicología de la Universidad Federal de Ceará (PPGP-UFC), con sus especificidades, abordando sus aspectos históricos, su estructura en términos de líneas de investigación y temas, proyectos y acciones de intercambios nacionales e internacionales, impactos sociales y su relevancia socio-académica, así como los desafíos que se presentan. El programa busca articular la práctica de la investigación con los desarrollos derivados de la extensión universitaria, ya que prioriza en su política de producción de conocimiento la tematización de los fenómenos sociales que se presentan, especialmente en el contexto de la Región Nordeste de Brasil. El texto señala, todavia, que esta especificidad constitutiva del Programa ha contribuido tanto a la consolidación de los laboratorios vinculados a las líneas de investigación del PPGP-UFC, como a su inserción social volcada al desarrollo local y regional. Finalmente, se indican los principales desafíos identificados en el contexto actual.

Palabras-clave: psicologia; investigación; extensión; noreste. 
Este artigo tem o objetivo de apresentar especificidades do Programa de Pós-Graduação em Psicologia da Universidade Federal do Ceará (PPGP-UFC), relacionando-as aos seus aspectos históricos, à sua relevância socioacadêmica e aos seus desafios atuais. Ao longo do texto, assinalou-se como sua política de produção de conhecimento se caracteriza, por se dedicar às problematizações sociais prementes no contexto nordestino, articulando a prática da pesquisa a ações de extensão universitária e propiciando a consolidação e ampliação das contribuições do programa ao desenvolvimento local e regional.

A elaboração deste texto teve como métodos de construção o levantamento e a análise da documentação disponível no PPGP-UFC, com o objetivo de articular a sua constituição com os desdobramentos advindos da prática extensionista, que sempre foi a peculiaridade do Departamento de Psicologia da UFC. Na sua proposição, foram revisitados projetos de criação e relatórios anuais dos núcleos e laboratórios de extensão, bem como a documentação relativa à Plataforma Sucupira e à Avaliação de Propostas de Cursos Novos (APCN), submetida à Coordenação de Aperfeiçoamento de Pessoal de Nível Superior (CAPES), que deu origem ao programa de pós-graduação.

A partir dos critérios de pertinência, circunstancialidade e representatividade histórica foi possível confrontar e verificar a documentação e tomá-la como referente da articulação da experiência extensionista como base para a constituição das práticas de pesquisa e consolidação do programa no contexto social de sua inserção.

O histórico do PPGP-UFC tem uma peculiaridade que merece ser recuperada para melhor compreensão do seu surgimento apenas três décadas após a criação do curso de graduação em Psicologia da UFC, cuja primeira turma ingressou em 1974. O curso de graduação tinha como prioridade as atividades de extensão universitária e, no decorrer dessas décadas, os docentes foram se agrupando e formando núcleos e laboratórios com intensa atuação nas comunidades. $\mathrm{Na}$ década de 1990, com a renovação do corpo docente do Departamento de Psicologia em razão de várias aposentadorias, iniciou-se um movimento para qualificação dos professores com vistas à criação da pós-graduação. Após o retorno de muitos professores com doutorado concluído, havia então condições para se propor a criação de uma pós-graduação stricto sensu, tendo sido formada uma comissão para elaboração da proposta, que foi aprovada na UFC e pela CAPES, iniciando-se, assim, o curso de mestrado em Psicologia em 2003.
Em 2013, quando o mestrado em Psicologia na UFC já estava consolidado, foi encaminhado à Avaliação das Propostas de Cursos Novos (APCN) o projeto para criação do curso de doutorado, o qual obteve aprovação da CAPES com nota 4. Dessa forma, deu-se em 2014 a criação do referido curso, que teve sua primeira turma de doutorandos em 2015. Constituído, portanto, dos cursos de mestrado e doutorado, o programa tem por objetivo geral formar pesquisadores e docentes qualificados para o exercício de atividades de pesquisa e ensino em Psicologia, bem como contribuir com a formação de profissionais de excelência para essa área, que atentem nas necessidades da população nordestina e brasileira em geral, decorrentes de suas condições de vida. Atualmente, o programa conta com 17 professores permanentes e três colaboradores, construindo-se como o único curso de mestrado acadêmico e de doutorado em Psicologia em universidade pública no Ceará. No Nordeste, são apenas nove cursos de doutorado em Psicologia em universidades públicas, o que atesta a importância do programa para essa região.

O PPGP-UFC possui três linhas de pesquisa, a saber: 1) Teorias e Práticas da Psicanálise: propõe-se a realizar estudos da dimensão inconsciente, dos processos de constituição psíquica e da sociabilidade humana, a partir da discussão de aspectos teóricos, metodológicos e clínicos da Psicanálise. Compreende também estudos envolvendo o diálogo da Psicanálise com as Ciências Humanas e a Saúde, o campo da Cultura e seus desdobramentos. É formada por quatro professores permanentes, sendo um deles pesquisador do CNPq; 2) Sujeito e Cultura na Sociedade Contemporânea: investiga, a partir de diferentes matrizes teóricas, a produção do sujeito na sua relação com a cultura, discutindo os campos da clínica, da educação e da política, na sociedade contemporânea. Priorizam-se os aspectos relativos ao laço social, à dominação cultural, ao sofrimento psíquico e às estratégias de resistências individuais e coletivas frente às novas tecnologias, ao consumo, à midiatização da sociedade, à compressão do tempo e à medicalização da vida. A linha é composta por cinco professores permanentes, sendo um deles bolsista de produtividade do CNPq e outra bolsista de produtividade da Fundação Cearense de Apoio ao Desenvolvimento Científico e Tecnológico (Funcap), além de dois docentes colaboradores; 3) Processos Psicossociais e Vulnerabilidades Sociais: congrega, sob diferentes perspectivas teórico-metodológicas em Psicologia Social, pesquisas acerca de situações de

Estudos de Psicologia, 24(2), abril a junho de 2019, 147-158 
vulnerabilidade social e dos processos psicossociais que lhes são concernentes, em suas interfaces com trabalho, organizações, pobreza, juventudes, violências, relações socioambientais e políticas públicas. Seus estudos envolvem, direta ou indiretamente, grupos em condições de desigualdades sociais, com ênfase na transformação social. A linha é formada por oito professores permanentes, sendo dois deles pesquisadores do $\mathrm{CNPq}$, além de uma docente colaboradora.

Até o ano de 2018 , foram produzidas duas coletâneas pelo programa: uma sobre as experiências de formação para a docência (Pascual, Ximenes, Moura Jr., \& Melo, 2012) e outra que abordou as diversas temáticas e pesquisas desenvolvidas pelo PPGP-UFC a partir de parcerias entre docentes e discentes em torno da questão do sujeito e das subjetividades contemporâneas (Lima, Germano, Sabóia, \& Freire, 2018).

Nas próximas seções, serão abordados os temas e as investigações que cada linha de pesquisa tem desenvolvido, enfocando as peculiaridades no processo de produção de conhecimento no programa. Em seguida, serão as contribuições do PPGP-UFC ao contexto local e regional. Por fim, alguns dos principais desafios serão frisados, levando-se em consideração exigências advindas do processo de internacionalização e disparidades regionais.

\section{Temáticas e investigações atualmente desenvolvidas pelas linhas de pesquisa do programa}

As pesquisas realizadas nas três linhas do programa guardam uma especificidade relacionada à sua forte articulação com projetos e demais ações de extensão ligados ao Departamento de Psicologia da UFC, o qual, ao longo de seus 45 anos de existência, tem sido reconhecido por sua tradição extensionista. Isso faz com que a relação entre universidade e comunidade também seja a tônica do programa de pós-graduação desde sua criação. Tal especificidade é realçada em função de que as problemáticas psicossociais que são enfrentadas na atuação extensionista são também fomentadoras de questões de pesquisa no programa, cujo aprofundamento teórico e científico, por sua vez, respalda a compreensão e construção de conhecimentos acerca dos processos de subjetivação na sociedade contemporânea, a partir de diferentes matrizes teórico-metodológicas.

Essa especificidade concernente à articulação das pesquisas desenvolvidas pelo programa nas ações de extensão reflete-se no fato de que diversos núcleos e laboratórios do Departamento de Psicologia da UFC, que se caracterizam pelo trabalho integrado entre ensino, pesquisa e extensão, estão ligados às linhas de pesquisa do PPGP-UFC. Como exemplo disso, a linha Teorias e Práticas da Psicanálise abriga o Laboratório de Psicanálise da UFC, o Núcleo de Estudos sobre Drogas (Nuced) e o Programa Clínica, Estética e Política do Cuidado. Articulados à linha Sujeito e Cultura na Sociedade Contemporânea estão o Laboratório de Psicologia em Subjetividade e Sociedade (Lapsus) e o Grupo Interdisciplinar de Estudos, Pesquisas e Intervenções em Psicologia Social Crítica (Paralaxe). Por sua vez, ligados à linha Processos Psicossociais e Vulnerabilidades Sociais estão o Núcleo Cearense de Estudos e Pesquisas sobre a Criança (Nucepec), o Núcleo de Psicologia Comunitária (Nucom), o Núcleo de Psicologia do Trabalho (Nutra), o Laboratório de Psicologia Ambiental (Locus), o Laboratório Cearense de Psicometria (Lacep), o Grupo de Pesquisas e Intervenções sobre Violência, Exclusão Social e Subjetivação (Vieses) e a Rede Internacional de Estudos e Pesquisas sobre Empreendedorismo e Liderança (Rinepe).

A tabela 1 apresenta a distribuição dos docentes por linha de pesquisa e núcleo ou laboratório de pertença.

Tabela 1. Distribuição dos Docentes Permanentes por Linhas e Laboratórios

\begin{tabular}{|c|c|}
\hline Linhas de Pesquisa/Docentes & Laboratórios e Núcleos \\
\hline \multicolumn{2}{|c|}{ Linha 1 - Teorias e Práticas da Psicanálise } \\
\hline $\begin{array}{l}\text { Caciana Linhares Pereira } \\
\text { Karla Patrícia Holanda Martins } \\
\text { Laéria Beserra Fontenele }\end{array}$ & $\begin{array}{l}\text { Laboratório de Psicanálise } \\
\text { da UFC }\end{array}$ \\
\hline Ricardo Pimentel Méllo & Nuced \\
\hline $\begin{array}{l}\text { Karla Patrícia } \\
\text { Holanda Martins }\end{array}$ & $\begin{array}{l}\text { Clínica, Estética e Política } \\
\text { do Cuidado }\end{array}$ \\
\hline \multicolumn{2}{|c|}{ Linha 2 - Sujeito e Cultura na Sociedade Contemporânea } \\
\hline $\begin{array}{l}\text { Idilva Maria Pires Germano } \\
\text { Luciana Lobo Miranda } \\
\text { Pablo Severiano Benevides }\end{array}$ & Lapsus \\
\hline $\begin{array}{l}\text { Aluísio Ferreira de Lima } \\
\text { Deborah Christina Antunes }\end{array}$ & Paralaxe \\
\hline \multicolumn{2}{|c|}{ Linha 3 - Processos Psicossociais e Vulnerabilidades Sociais } \\
\hline Antônio Caubi Ribeiro Tupinambá & Rinepe \\
\hline Cássio Adriano Braz de Aquino & Nutra \\
\hline $\begin{array}{l}\text { James Ferreira Moura Junior } \\
\text { Verônica Morais Ximenes }\end{array}$ & Nucom \\
\hline João Paulo Pereira Barros & Vieses \\
\hline Veriana de Fátima Rodrigues Colaço & Nucepec \\
\hline Walberto Silva dos Santos & Lacep \\
\hline Zulmira Áurea Cruz Bomfim & Locus \\
\hline
\end{tabular}


Isso tem contribuído tanto para a consolidação dessas linhas, quanto para a articulação da pós-graduação à graduação em Psicologia na UFC. Além disso, tem favorecido a formulação de propostas de investigação capazes de questionar os problemas sociais e respondê-los em consonância com os desafios da ciência contemporânea. O Brasil ainda tem a marca de ser extremamente desigual e com graves problemas, tais como: enormes bolsões de pobreza; altas taxas de violência sofridas por mulheres, por negros e por pessoas LBGTI; alarmantes índices de assassinatos e prisões de jovens moradores de periferias; e trabalhos precarizados, alguns (especialmente em áreas rurais) assemelhando-se ao trabalho escravo. Diante disso, importa que as investigações e produções científicas do programa busquem problematizar tais situações e construam conhecimentos que subsidiem o campo da Psicologia, que, não poucas vezes, ignora essas realidades sociais desiguais. Assim, seja em projetos "guarda-chuvas" de docentes, seja nas pesquisas de dissertações e teses de discentes, emergem daí o envolvimento e o compromisso com mudanças em relações injustas geradoras de sofrimento psíquico, ao mesmo tempo que se buscam ações favorecedoras da convivência com as diversidades (raciais, de gênero etc.). Para ilustrar, de forma sintética e geral, são elencados a seguir alguns temas e projetos coordenados pelos docentes em cada uma das linhas de pesquisa a partir de sua inserção em laboratórios e núcleos.

A linha Teorias e Práticas em Psicanálise realiza pesquisas e estudos que se interrogam a respeito do exercício da prática clínica, quanto a seus fundamentos, conceitos e teorias em espaços tradicionais e não tradicionais, em busca da presença da Psicanálise em dispositivos variados, em articulação com os campos da Saúde e da Educação. Destacam-se pesquisas que relacionam Psicanálise, Cultura, Educação e Literatura (Fontenele \& Ducerisier, 2017; Linhares, 2018), estudos sobre os possíveis efeitos da experiência da fome em processos de subjetivação de mães e crianças em situação de desnutrição e pesquisas sobre a utilização do diagnóstico psiquiátrico e da medicalização de crianças nos serviços públicos de saúde (Aguiar, Martins, \& Rosa, 2019). Além disso, há pesquisas que discutem a ampliação das práticas de cuidado em saúde (Méllo, 2018), relacionadas ao uso de substâncias lícitas e ilícitas, em territórios diversos, como: zonas de prostituição, equipamentos de saúde (CAPS-ad), pessoas em situação de rua, adolescentes que desafiam a lei e são reclusos em sistemas socioeducativos.
A linha Sujeito e Cultura na Sociedade Contemporânea tem como foco o desenvolvimento de pesquisas sobre a inserção da Psicologia no debate crítico e ético da cultura tardomoderna, discutindo as diversas condições que afetam as subjetividades contemporâneas (Severiano, 2017; C. M. Silva, Freire, \& Acselrad, 2019), tais como a cultura do consumo, os processos identitários e de reconhecimento, a mídia, as novas tecnologias e as novas formas de controle e regulação social no capitalismo tardio. Atualmente, as pesquisas coordenadas pelos docentes permanentes da linha envolvem a desnaturalização da aplicação de diagnósticos, dos enquadramentos e da medicalização de forma instrumental e desconectada das condições de existência das pessoas (Lima \& Ciampa, 2017). Discutem, ainda, questões relativas à educação, aos modos de subjetivação e à micropolítica no cotidiano escolar (Miranda, Oliveira, Souza Filho, \& Sousa, 2018). Além disso, são problematizadas questões sobre tecnologia digital e subjetividade (Antunes \& Maia, 2018), vigilância, monitoramento e práticas de controle contemporâneas (Benevides, 2018) e narrativas autobiográficas femininas (Germano, Carneiro, Pontes, Silva, \& Gomes, 2018).

No que se refere à linha Processos Psicossociais e Vulnerabilidades Sociais, encontram-se em andamento pesquisas "guarda-chuvas", dissertações e teses que abordam questões como as implicações psicossociais da pobreza, compreendendo esse fenômeno sob uma perspectiva multidimensional (Ximenes, Nepomuceno, Cidade, \& Moura Jr., 2016); violência urbana envolvendo segmentos juvenis em contextos periféricos de Fortaleza, sob a perspectiva de jovens negros, familiares de jovens assassinados ou privados de liberdade e profissionais de políticas públicas, a partir de uma orientação de pesquisa participativa, o que se faz socialmente relevante pelo fato de que o Ceará se apresenta como o estado brasileiro com o maior índice de homicídio na adolescência (Barros, Benicio, Silva, Santos, \& Torres, 2017; Menezes, Colaço, \& Adrião, 2018); fatores psicossociais relativos ao comportamento criminal (W. S. Santos, Holanda, Meneses, Luengo, \& GomezFraguela, 2019); processos laborais, precarização do trabalho e flexibilização do tempo (Oliveira, Moita, \& Aquino, 2016); processos socioambientais, afetos e subjetividade (H. G. S. Silva, Bomfim, \& Costa, 2019); relações entre pobreza, bem-estar e processos de discriminação (Moura Jr. \& Sarriera, 2017); e avaliação psicológica (Cardoso \& Silva-Filho, 2018). 


\section{Contribuições sociais e acadêmicas em âmbito local e regional}

Em 2019, o programa conta com 72 alunos matriculados no doutorado e 49 no mestrado. Embora a maior procura de candidatos seja oriunda das regiões Nordeste e Norte, o PPGP continua recebendo inscrições de diferentes regiões do país. O programa conta também com a experiência advinda de estudantes convênio de estudantes de pós-graduação PEC PG, atividade atendida pelo acordo de Cooperação Educacional, Cultural ou de Ciência e Tecnologia firmado entre o Brasil e outros países em desenvolvimento. Assim, em seus dois níveis - mestrado e doutorado vem-se seguindo o propósito de ampliar as oportunidades de formação acadêmica, primando pelo compromisso de priorizar investigações criticamente implicadas com a realidade social do país, particularmente a do Nordeste. Até junho de 2019, o PPG em Psicologia da UFC formou mais de 300 mestres, além de quatro teses de doutorado terem sido defendidas e oito pós-doutorados foram realizados. A série histórica do número de ingressos no programa, desde a sua abertura até os dias atuais, pode ser observada na Figura 1.

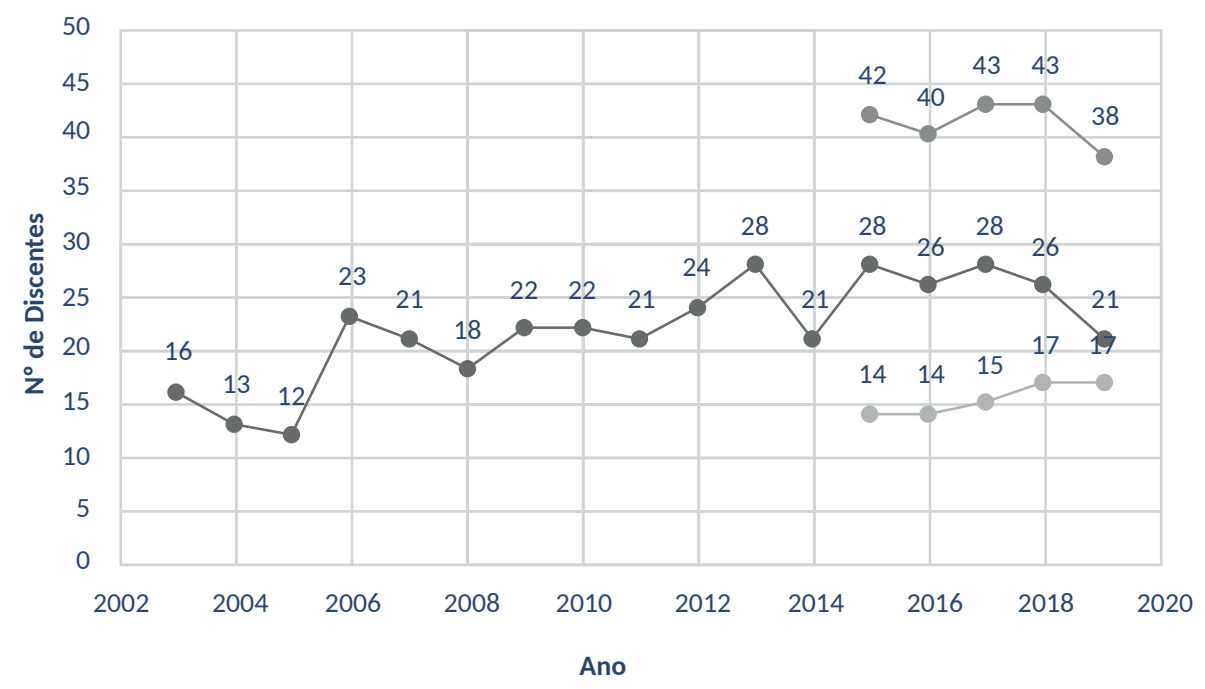

- Mestrado

- Mestrado e Doutorado

Figura 1. Evolução do Número de Ingressos no Programa.

Já o acompanhamento de egressos tem sido uma das ações sistemáticas utilizadas para orientar as avaliações e os planejamentos do programa, em função da relevância que tem essa informação, como elemento revelador da qualidade da formação oferecida, pois reflete a efetivação de seus objetivos em termos do perfil esperado dessa formação. Foram focados especialmente dois aspectos: um relativo à continuidade dos estudos dos egressos em nível de doutorado e outro sobre o ingresso ou a continuidade em atividades de docência em nível superior. Com os dados disponíveis atualmente, ao se cruzarem questionários respondidos pelos egressos e seus currículos lattes, é possível fazer uma estimativa de que $18 \%$ dos egressos do mestrado já obtiveram o título de doutor e $20 \%$ estão cursando doutorado ( $77 \%$ desses cursam no programa).
No que concerne à realização de atividades de docência de nível superior, destaca-se que 52\% dos egressos do PPG em Psicologia da UFC encontram-se no exercício do magistério, tanto em instituições de ensino superior públicas quanto em privadas do estado. Nas primeiras, estão lotados em campi da UFC na capital e no interior do Ceará, ou mesmo em universidades públicas de estados do Nordeste, Norte e de outras regiões brasileiras. No segundo caso, abrange principalmente a capital e alguns municípios mais populosos do Ceará, tais como Sobral, Quixadá, Crato e Juazeiro do Norte.

Cientes da responsabilidade na formação de professores e pesquisadores em outras regiões no estado do Ceará, o PPG em Psicologia da UFC assessorou diretamente a criação do Programa de Mestrado Profissional em Psicologia e Políticas Públicas, aprovado pela CAPES em 2018 e vinculado ao curso de Psicologia 
da UFC do campus de Sobral. Dos oito professores que integram o corpo docente do mestrado profissional, cinco são egressos do curso de mestrado do PPGP. Esse regime de colaboração com mestrado profissional e com a graduação da cidade de Sobral corrobora a política de formação do programa, que insere egressos na docência em instituições de ensino superior (IES) e apresenta parcerias institucionais, contribuindo para a influência do PPGP na região. Inclusive a vinculação do programa com a interiorização universitária motivou a criação do curso de Psicologia na cidade de Sobral (CE) em 2006, a partir de demanda da Reitoria da UFC, dentro do horizonte do Programa de Reestruturação e Expansão das Universidades Federais (Reuni). Nesse caso, um grupo de professores da pós-graduação foi responsável pela elaboração do projeto de criação do primeiro curso público de Psicologia e primeiro mestrado profissional em Psicologia fora da capital.

Ainda sob esse aspecto, existem relações de cooperação entre grupos de pesquisa do programa e grupos de outras instituições, a exemplo da articulação entre o Vieses e o reaPODERE, da Unilab, no desenvolvimento das análises da etapa quantitativa da pesquisa "Juventude e violência urbana: cartografia de processos de subjetivação na cidade de Fortaleza-CE", financiada pela Chamada Universal 01-2016, do CNPq, e que conta também com o apoio da Assembleia Legislativa do Estado do Ceará e do Unicef, por meio do Comitê Cearense pela Prevenção de Homicídios na Adolescência. Destaca-se também a parceria entre o Nutra e o Grupo de Trabalho, Movimentos Sociais e Políticas Públicas (Trampos), da USP, nos estudos sobre as novas configurações do cenário laboral. O Paralaxe, por sua vez, tem desenvolvido parcerias com o Laboratório de Estudos de Crítica Social, Direitos Humanos e Intersubjetividade (Incidir), do Programa de Pós-Graduação em Psicologia Social do Instituto de Psicologia da Universidade de São Paulo (IPUSP), e com o Núcleo de Estudos e Pesquisas em Desenvolvimento Humano, Psicologia Educacional e Queixa Escolar (Psiqued), do Programa de Pós-Graduação em Psicologia da Universidade Federal do Piauí (UFPI). Entre as colaborações recentes destaca-se a participação no projeto "Coisas frágeis: narrativas sobre experiências de sofrimento e os efeitos dos enquadramentos psi", desenvolvido no Paralaxe. Ressalta-se, ainda, a parceria do Núcleo de Psicologia Comunitária (Nucom/UFC) com o Grupo de Pesquisa em Psicologia Comunitária (GPPC/UFRGS) no desenvolvimento de pesquisa conjunta intitulada "Impactos da pobreza no desenvolvimento da saúde comunitária: avaliação psicossocial das comunidades do Bom Jardim (Fortaleza) e da Canafístula (Apuiarés/Ceará)", concluída em 2013 e contemplada pelo Edital MCTI/CNPq/MEC/CAPES 07/2011. Ressalta-se também a parceria entre o Núcleo de Estudos sobre Drogas (Nuced/UFC), a Prefeitura de Fortaleza e a Fundação Oswaldo Cruz (Fiocruz) para a realização de pesquisa sobre o acompanhamento da população em situação de rua em Fortaleza. A realização de projeto de pesquisa conjunto, integrando Psicologia Social e Psicologia Ambiental entre o Laboratório de Psicologia Ambiental (Locus-UFC) e o Grupo de Pesquisa do Núcleo de Estudos da Exclusão/Inclusão Social (Nexin), do Programa de Pós-Graduação da PUC/SP, faz parte dessas articulações interinstitucionais.

Em relação à inserção social do programa, ela tem ocupado historicamente espaço relevante $\mathrm{e}$ direcionador no Departamento de Psicologia da UFC na consolidação de articulações teórico-práticas da pesquisa com o ensino e a extensão. Essas atividades envolvem: cursos, seminários, organização de congressos nacionais e internacionais voltados para a comunidade acadêmica em geral e grupos específicos, como jovens, estudantes de ensino médio de escolas públicas, associações comunitárias e populações em situações de vulnerabilidade social; intervenções em escolas da educação básica, comunidades, associações, órgãos públicos; participação de membros do programa em comissões e conselhos de saúde e direitos humanos, prevenção da violência e educação básica, tais como: Conselho Consultivo do Comitê Cearense pela Prevenção de Homicídios na Adolescência (Unicef e Assembleia Legislativa); Rede Territorial para Prevenção de Homicídios na Infância, Adolescência e Juventude do Grande Bom Jardim, região da periferia de Fortaleza com as maiores taxas de homicídios juvenis; Fórum Popular de Segurança Pública do Ceará; Fórum Cearense de Luta Antimanicomial; Conselho Estadual de Saúde do Ceará (Cesau); Conselho Estadual de Educação do Ceará; Movimento Cada Vida Importa: a universidade na prevenção e no enfrentamento à violência no Ceará (MCVI); Diretoria da ANPEPP, entre outros.

Essas participações ativas, estabelecendo relação entre movimentos sociais e a constituição de políticas públicas, ampliam o espaço de atuação da universidade para além das suas fronteiras físicas, fazendo com que o trabalho acadêmico ganhe respeito e importância por diversos segmentos da sociedade civil organizada que, 
com apoio das pesquisas do programa, atua junto ao Estado para a criação e execução de políticas públicas pertinentes às suas demandas, como os movimentos sindicais da classe trabalhadora, o movimento estudantil, as ONGs, os movimentos de mulheres, de trabalhadores rurais, do negro, dos povos indígenas etc. Desse modo, como apontou B. S. Santos (2014), fortalecem-se as experiências que apostam em formas de fazer pesquisa, que não perpetuem a construção de saberes afeitos à colonialidade imperante, que só reproduz relações autoritárias por mecanismos de exploração, exclusão e opressão.

O envolvimento do programa em diversas ações que, por exemplo, mobilizam adolescentes e jovens, profissionais e gestores públicos, difundindo dados de investigações acadêmicas e favorecendo discussões sobre direitos humanos e cuidados em saúde, promove a elaboração de políticas públicas para a prevenção de violências letais sofridas por jovens, mulheres, indígenas, populações de rua, comunidades rurais, entre outros. Assim, também fomentam-se, por exemplo, o enfrentamento do combate ao racismo e a redução de desigualdades sociais que repercutem no sofrimento psíquico dessas populações.

É o caso do projeto "Histórias desmedidas" que, derivando de pesquisas desenvolvidas pelo Vieses-UFC, enfoca trajetórias e perspectivas de vida de adolescentes em cumprimento de medida socioeducativa e de jovens privados de liberdade, o qual, de 2016 a 2018, realizou 14 oficinas em um Centro de Referência Especializada da Assistência Social (Creas), na periferia de Fortaleza, atingindo um número de 40 adolescentes e jovens. Já o projeto "EntreTantos", ligado também ao Vieses-UFC, segue também essa linha ao se voltar para o mapeamento de práticas de resistências juvenis às violências nas periferias urbanas. $O$ projeto desenvolveu, em 2018, 11 rodas de conversas com um coletivo de adolescentes e jovens chamado Estrelas do Campo e 20 oficinas com mulheres vítimas de violência na Comunidade Santa Filomena, ambos na região do Jangurussu, periferia da capital cearense. As intervenções desses dois projetos de extensão deram ressonância aos resultados da pesquisa "Juventude e violência urbana: cartografia de processos de subjetivação na cidade de Fortaleza-CE", ao mesmo tempo que retroalimentaram as pesquisas de mestrandos e doutorandos do programa que participam do Vieses-UFC.

Tem se tornado uma prática no programa, para o enfrentamento das vulnerabilidades psicossociais, a realização de oficinas com devolutiva de pesquisas. $\mathrm{Na}$ investigação interestadual "Impactos da pobreza no desenvolvimento da saúde comunitária: avaliação psicossocial de comunidades rurais das regiões Nordeste, Norte e Sul", foram realizadas reuniões na Unidade Básicas de Saúde (UBS) de Juvinópolis e do Rio do Salto no município de Cascavel (Paraná), com a presença de profissionais do Centro de Referência da Assistência Social (Cras). Foram distribuídas cartilhas com o resultado e a discussão da pesquisa e a situação atual de cada comunidade. Contou também com a participação de professores e estudantes da Universidade Paranaense (Unipar), instituição parceira da pesquisa.

Outro exemplo refere-se à educação básica, em que se destaca o Programa de Extensão TVEZ, do Laboratório de Psicologia em Subjetividade e Sociedade (Lapsus), que articula os cursos de Publicidade, Jornalismo e Psicologia, com o objetivo de promover o uso crítico das mídias por meio de ações integradas em escolas da rede pública de Fortaleza e região metropolitana. Nessa mesma direção, há o trabalho desenvolvido pelo Laboratório de Pesquisa em Psicologia Ambiental (Locus) na Escola Ita-Ara, da etnia Pitaguarys, em Monguba, Pacatuba-CE, com o objetivo de desenvolver atividades referentes à preservação de povos originários, tendo como público-alvo adolescentes e jovens indígenas e não indígenas. Além disso, o projeto de extensão "Re-Tratos da juventude", ligado ao Vieses, tem sido um campo de pesquisas de dissertações e teses de discentes do programa em escolas públicas da periferia de Fortaleza acerca de questões concernentes às relações entre juventude, violência e produção de subjetividades.

Outra atividade de colaboração entre docentes do programa refere-se à parceria de pesquisa e extensão na Faculdade Católica Rainha do Sertão, situada no município cearense de Quixadá, que tem contribuído para a ampliação da rede de cuidados clínicos a crianças e adolescentes em situação de sofrimento psíquico grave, especialmente nos municípios de Quixadá e Quixeramobim, em um cenário que se caracteriza por acentuadas desigualdades sociais e pela ausência de políticas de intervenção e tratamento, tendo como consequência a intensa medicalização de crianças e jovens. O projeto articula-se ao programa de extensão "Cuidado, estética e política".

Por fim, tem-se o projeto de pesquisa "Práticas de cuidado e promotoras de saúde", que se articula a duas ações de extensão, uma relacionada à utilização do guia Gestão Autônoma da Medicação (GAM), 
em um CAPS-ad na cidade de Fortaleza, contribuindo para incluir neste equipamento de saúde discussões sobre o uso de medicamentos, ao mesmo tempo que esclarece que as pessoas acompanhadas se responsabilizem pelo cuidado de si, assumindo ativamente seus tratamentos de saúde. A outra ação refere-se ao projeto de extensão e pesquisa "Cuida! Práticas integradas de cuidado com profissionais do sexo da Barra do Ceará", que teve início em 2015, a partir da aproximação do Núcleo de Estudos sobre Drogas (Nuced), com educadores e profissionais do Centro Urbano de Cultura, Arte, Ciência e Esporte (Cuca), equipamento da Prefeitura de Fortaleza, localizado na Barra do Ceará, e Agentes Comunitárias de Saúde do Posto de Saúde Lineu Jucá, voltadas ao cuidado em saúde de trabalhadora do sexo. As estratégias de Redução de Danos (RD) têm sido a principal ferramenta nessa ação, pois potencializam o enfrentamento de preconceitos e moralismos que circunscrevem a prostituição (trabalho historicamente estigmatizado, sobretudo pelas relações desiguais de gênero e pela culpabilização da mulher pelo exercício de sua sexualidade).

Vale salientar que todas as atividades de inserção social abordadas acima ocorrem articuladamente entre professores e estudantes de graduação e de pós-graduação, entrelaçando o tripé acadêmico da pesquisa, do ensino e da extensão. Por fim, como derivação desse tripé acadêmico no PPGP-UFC, são promovidos sistematicamente desde aulas inaugurais, seminários de pesquisa e de tese, palestras e cursos de extensão, abordando temáticas diversas, como política de pesquisa participativa em Psicologia, gênero e interseccionalidade, racismo, saúde mental e práticas de cuidado, prevenção de violência contra jovens, clínica e intervenção precoce, até a situação de pessoas moradoras de rua, desafios das políticas públicas, estigma em sala de aula, entre outros. Essas atividades mostram uma inclinação do programa para a problematização de questões envolvendo políticas de vulnerabilidade social e seus diversos efeitos, a partir de diferentes prismas teórico-metodológicos.

Também no tocante às contribuições acadêmicas do PPGP-UFC ao fortalecimento de ações acadêmicas na região Nordeste, vale destacar dois eventos internacionais realizados na cidade de Fortaleza e presididos por docentes permanentes do seu Colegiado: a $5^{\text {a }}$ Conferência Internacional de Psicologia Comunitária ( $5^{\text {a }} \mathrm{CIPC}$ ) e o VII Simpósio Internacional sobre Juventude Brasileira (VII Jubra). A $5^{\text {a }}$ CIPC, que teve como tema "Psicologia comunitária no mundo atual: desafios, limites e fazeres", foi realizada no período de 3 a 6 de setembro de 2014 com a parceria entre o Programa de Pós-Graduação em Psicologia da UFC e a Universidade de Fortaleza (Unifor). O evento contou com 1.553 inscritos, evidenciando a grande procura das pessoas por conhecimento em Psicologia Comunitária e por espaços para compartilhar seus trabalhos, encontrar novas informações e construir parcerias. Participaram do evento 946 pessoas, sendo 769 brasileiras e 177 estrangeiras, abrangendo 35 países. Teve também uma representatividade considerável de estudantes de pós-graduação, tanto em nível de mestrado quanto de doutorado, bem como de pesquisadores de inúmeras instituições brasileiras e estrangeiras, totalizando 625 participantes envolvidos com o sistema de pós-graduação apresentando suas investigações e participando de discussões promovidas pelo evento. A partir das conferências apresentadas, foi organizado um livro com o tema do evento (Ximenes, Sarriera, Bomfim, \& Alfaro, 2016).

Já em 2017, foi organizado o VII Jubra, com o tema "Juventudes em movimento: experiências, redes e afetos", que teve 2.169 inscritos e número estimado de 1.300 participantes de todas as regiões do Brasil e também de pesquisadores estrangeiros. Além de congregar pesquisadores e professores brasileiros e de outros países, tratou-se de um evento científico que contou com a participação de profissionais, estudantes, jovens, gestores públicos e agentes comunitários para a discussão de pesquisas, programas e projetos sociais referentes às juventudes e a seus modos de vida em diferentes contextos e condições. O evento teve apoio da CAPES e o mérito da criação da Redejubra, com estatuto e Conselho Diretor, que se propôs a formar uma rede nacional interdisciplinar de pesquisadores interessados na temática da juventude brasileira, gerando também uma publicação com pesquisadores nacionais e estrangeiros (Colaço, Germano, Miranda, \& Barros, 2019).

\section{Desafios de um Programa fora do eixo sul-sudeste no contexto atual}

Em meio aos crescentes cortes na educação, que vêm atingindo inclusive os programas de pós-graduação, manter a qualidade da formação de pesquisadores e docentes numa instituição pública, fora do eixo Sul-Sudeste, tem sido um empreendimento desafiador. A perspectiva de Reforma da Previdência como meta prioritária do atual governo fez com que aumentassem os pedidos de aposentadoria de docentes na UFC, inclusive 
de professores permanentes do quadro. Por outro lado, o intenso trabalho do cotidiano das pós-graduações, com constantes aumentos dos índices de produtividade, apresenta-se como um desafio aos candidatos docentes que querem ingressar no quadro de permanentes no PPGP. Essa dificuldade particularmente atinge o programa, por ter inserção importante no curso de graduação, com participação ativa em núcleos e laboratórios que desenvolvem pesquisa e atividades de extensão a eles correlacionadas. Diante dessa conjuntura, a renovação de docentes apresenta-se como um desafio.

Também no que se refere à internacionalização, são encontrados desafios relacionados ao limitado financiamento de bolsas e ao incentivo de pós-doutoramento de professores no exterior. Diante das dificuldades, nos últimos anos, o programa tem fortalecido sua rede de relações com instituições de ensino no eixo Sul-Sul, EUA e Europa. Alguns professores do programa têm estabelecido parcerias de pesquisa e outras atividades com professores de universidades estrangeiras, particularmente como desdobramento dos estágios de pós-doutorado realizados pelos docentes no exterior. Também há vinculações com instituições estrangeiras decorrentes de assessorias e representações estabelecidas entre os docentes do programa e as entidades científicas internacionais. $\mathrm{Na}$ Comunidade Europeia, estabeleceu-se intercâmbio com o Grupo de Investigación "Empleo, Género y Cohesión Social" (Egeco), da Universidad Complutense de Madrid, com o Grupo Pessoa Ambiente (Gripa), da Universidade da Coruña, além do intercâmbio de estudantes do Master en Intervención Psicosocial de la Universidad de Barcelona (UB), os quais fazem um estágio prático no programa. Desde 2014, também foi estabelecido um convênio de cooperação internacional entre França, Colômbia e Brasil (Prefalc), com o Insa, da Universidade de Lyon 2 em Lyon (França), Universidade Nacional da Colômbia (Unal) e UFC. Tem-se ainda em desenvolvimento um processo de doutoramento em cotutela com a Universidade de Nice e com a Universidade Livre de Bruxelas. Na Alemanha, no contexto organizacional e laboral, foi estabelecida uma parceria com a Universidade de Colônia, com a Universidade de Lüeneburg e com a Universidade de Heidelbelg, através de bolsas de pós-doutorado e doutorado sanduíche. Em Portugal, destaca-se a parceria com o Centro de Investigação e Estudos de Sociologia, do Instituto Universitário de Lisboa (CIES/IUL), para o desenvolvimento de pesquisas e estudos conjuntos acerca dos processos de mobilidade acadêmica e internacionalização da Educação e seus impactos na formação e inserção no mundo do trabalho. No eixo Sul-Sul, estabeleceu-se intercâmbio com o Programa de Fundamentos Históricos y Políticos de las Prácticas Psicológicas del Instituto de Fundamentos y Métodos en Psicología da Universidad de la Republica (Udelar/ Uruguay). No México, tem-se a parceria com grupos da Universidade Autónoma de Yucantán (México) e com a Rede Latino-Americana de Formação em Psicologia Comunitária, que reúne mais de 21 universidades. A parceria do programa com a Universidade Autónoma de Yucantán (México) está relacionada a trabalhos desenvolvidos em Psicologia Comunitária e especificamente ao desenvolvimento de uma pesquisa em parceria sobre as implicações psicossociais da pobreza, desenvolvida no Ceará desde 2011 e em Yucatán desde 2014. Em 2017, foi criada a "Red de Estudios Transculturales de la Pobreza", que tem como objetivo o desenvolvimento de pesquisas, estudos e intercâmbios sobre a pobreza na Universidad Autónoma de Yucatán (Uady), no Núcleo de Psicologia Comunitária da Universidade Federal do Ceará (UFC) e na Universidade Católica da Colômbia (UCC). O convênio com a Colômbia se estende à Universidad Católica de Colombia (Bogotá) e Universidad de Antióquia (Medelin) a partir do desenvolvimento de uma pesquisa em parceria sobre as implicações psicossociais da pobreza e sentimento de comunidade com os dados comparativos do Brasil e da Colômbia. Nos Estados Unidos da América, a partir de 2017, iniciou-se o estabelecimento de parceria com o Programa do Graduate Center da Cidade de Nova York (Cuny), mais particularmente no Programa de Pós-Graduação em Critical Social/ Personality Psychology. O convênio visa estabelecer possíveis conexões entre os princípios epistemológicos, éticos e metodológicos da pesquisa-intervenção desenvolvidos no âmbito da Psicologia Social no Brasil e as pesquisas com base na Critical Partipatory Action Research (CPAR), no Instituto The Public Science Project (PSP), no Centro de Pós-Graduação em Psicologia da Cuny. Ainda nos EUA, o Departament of Africana Studies da Brown University (Providence - Rhode Island-USA) foi o lócus de doutorado sanduíche para uma das alunas do programa, e outras formas de intercâmbio entre a Brown University e o PPGP da UFC estão sendo planejadas. Com o Canadá, são estabelecidos intercâmbios de pesquisa com a Simon Fraser University.

No ano de 2018, o programa obteve aprovação para execução de um projeto de internacionalização intitulado "Pesquisa participativa, inclusão social 
e política: sofrimento psíquico em segmentos vulnerabilizados", que conta com a participação de quatro professores externos, sendo três deles de instituições estrangeiras, uma professora associada com Agregação na Faculdade de Psicologia e de Ciências da Educação da Universidade do Porto, em Portugal; uma professora associada da School of Education da Saint Peter's University em Jersey City, New Jersey, nos Estados Unidos; e um professor da École Supérieure de Travail Social, Université Paris, Sorbonne, Paris IV, Paris, na França. O eixo de investigação foi definido pela sua transversalidade na abordagem de três temas presentes nas pesquisas dos professores e discentes, são eles: a) Saúde mental e sofrimento psíquico na educação inclusiva e no trabalho; b) Interseccionalidade: gênero, raça e classe; c) Juventudes, poder e resistência: o papel da mídia e das novas tecnologias nos processos de subjetivação na contemporaneidade em perspectiva crítica. Em março de 2019, deu-se início à execução da proposta, o que foi considerado um significativo passo para avançar no aspecto da internacionalização do programa.

Em estudo realizado acerca das estratégias empreendidas pelos programas de pós-graduação em busca da internacionalização (Ramos, 2018), foi denotada, entre os cursos de excelência no Brasil, fundamentalmente os alocados com avaliação 6 e 7 pela CAPES, a utilização da mobilidade acadêmica como mecanismo de criação de redes e colaborações internacionais.

Cientes das limitações que atravessam a constituição e busca da reafirmação do programa no cenário da pós-graduação brasileira, constituiu-se um esforço de estruturação das redes de pesquisa a partir de parcerias estabelecidas pelos docentes. Tal ação, embora pontual, abriu espaço para a construção de possibilidades que hoje são concretizadas através do procedimento do doutorado sanduíche com a participação dos alunos em instituições internacionais, bem como por meio da obtenção de apoio institucional para efetivação da colaboração de professores visitantes.

Para discussão e superação dos desafios acima elencados, uma das estratégias tem sido a solicitação de visita dos coordenadores de área da CAPES, bem como a participação e troca da realidade do PPGP nos seminários desenvolvidos por aquela agência de fomento. Outra forma desenvolvida é a realização semestral de planejamento estratégico em que se trabalha com planos e metas a curto, médio e longo prazo. Os desafios descritos, tais como a expansão da internacionalização e a renovação de professores, foram anteriormente identificados nesses encontros. No primeiro caso, como decorrência desses planejamentos, planos a curto prazo puderam ser colocados em prática: a candidatura do Edital de Professor Visitante, em que o PPGP pela primeira vez concorreu e foi contemplado; a participação de editais de doutorado sanduíche e de pós-doutorado - PNPD. A ampliação de submissões de propostas para bolsa PQ e também os editais para financiamentos de projetos de pesquisa têm sido outro investimento coletivo importante que corrobora com as metas do quadriênio em curso, inclusive com resultado positivo, como a conquista de uma nova bolsa $\mathrm{PQ}$, de modo que, atualmente, as três linhas contam com docente bolsista PQ. No segundo caso, visando suprir as aposentadorias, no ano de 2019, ainda em fase de submissão, abriram-se quatro vagas para professores permanentes com produção científica consolidada.

A médio prazo, objetiva-se o aumento da internacionalização das publicações, a ampliação e consolidação das parcerias internacionais acima elencadas. Para professores em parceria com estudantes, sobretudo aqueles inseridos em programas no exterior, são priorizadas publicações em língua estrangeira. As ações a curto e médio prazo têm como meta a longo prazo a consolidação do doutorado, visando à nota 5 do PPGP. Para tanto, em que pese a conjuntura macro desfavorável, caracterizada por contingenciamentos e o comprometimento da autonomia financeira das universidades públicas, faz-se necessário, mais do que nunca, o esforço conjunto na superação dos pontos frágeis.

Esse caminho inaugural, entretanto, não é suficiente para se garantir uma política de internacionalização sustentável, daí a necessidade de se empreender por meio da constituição de uma produção de conhecimento e intervenção críticos, que toma como referente o viés extensionista em associação à pesquisa, o nosso principal mecanismo de colaboração com a Psicologia a partir da realidade onde se atua. É esse esforço que possibilita o salto para uma compreensão de que a internacionalização não é uma atitude passiva diante de um conhecimento que advém de fora, mas o potencial de, a partir de uma interlocução com diversos outros saberes - não tomados hierarquicamente -, viabilização de um sentido mais pleno de cooperação internacional.

\section{Considerações finais}

A intenção deste artigo foi dar visibilidade a um programa de pós-graduação que tem como uma de suas principais peculiaridades o desdobramento de atividades de pesquisa em ações extensionistas. Tal aspecto 
amplia as contribuições sociais do programa, conectando a história de seu surgimento e o atual funcionamento de suas linhas de pesquisa.

Por estar situado no Nordeste brasileiro, o PPGP-UFC traz algumas características que ressaltam necessidades $\mathrm{e}$ desafios particulares. Em primeiro lugar, a necessidade de estar em sintonia com a realidade da desigualdade social brasileira, que na nossa região se torna mais intensa e exposta. Por sua vez, também a distribuição dos financiamentos reproduz essa desigualdade, comparando-se, em termos gerais, os programas de pós-graduação do Sul e Sudeste com os do Norte e Nordeste, o que impõe trabaIhar com recursos limitados e manter, ao mesmo tempo, a qualidade da formação e os índices de produção, bem como assegurar estratégias para conquistar níveis mais elevados na avaliação da CAPES, inclusive para a continuidade do seu credenciamento.

Diante desse quadro, o programa continua empenhado em manter a qualidade da formação crítica dos discentes e também em fazer avançar suas avaliações, na perspectiva de conquistar a nota 5 na CAPES, com a consolidação do curso de doutorado. Para tanto, tem sido necessário um esforço coletivo em ações de planejamento e estratégias direcionadas à superação dos pontos ainda frágeis.

O PPGP-UFC em seus eixos visa contribuir para o aperfeiçoamento de habilidades para a identificação de problemas relativos à constituição do sujeito em seu contexto social e para a superação de desigualdades presentes em tal contexto, transversalizando os campos da clínica, da saúde, da educação, dos direitos humanos, das novas tecnologias e da política na sociedade contemporânea.

\section{Referências}

Aguiar, G. M. R. D., Martins, K. P. H., \& Rosa, M. D. (2019). Criança, família e acolhimento institucional: entre a norma e a constituição psíquica. Configurações. Revista de Sociologia, (23), 90-104. doi: $10.4000 /$ configuracoes. 6900

Antunes, D. C., \& Maia, A. F. (2018). Big Data, exploração ubíqua e propaganda dirigida: novas facetas da indústria cultural. Psicologia USP, 29(2), 189-199. doi: 10.1590/0103-656420170156

Barros, J. P. P., Benicio, L. F. S., Silva, D. B., Santos, C. L., \& Torres, F. J.P. (2017). Homicídios juvenis e os desafios à democracia brasileira: implicações ético-políticas da psicologia. Psicologia: Ciência e Profissão, 37(4), 1051-1065. doi: 10.1590/1982-3703002892017

Benevides, P. S. (2018). Neoliberalismo, psicopolítica e capitalismo da transparência. Psicologia \& Sociedade, 29(e164064), 1-11. doi: 10.1590/1807-0310/2017v29164064
Cardoso, L. M., \& Silva-Filho, J. H.D. (2018). Satepsi e a qualidade técnica dos testes psicológicos no Brasil. Psicologia: Ciência e Profissão, 38(spe), 40-49. doi:10.1590/1982-3703000209112

Colaço, V. F. R., Germano, I. M. P., Miranda, L. L., \& Barros, J. P. (Orgs.). (2019). Juventudes em movimentos: experiências, redes e afetos. Fortaleza: Expressão Gráfica.

Fontenele, L., \& Ducerisier, F. (2017). Alain Didier-Weill: psychanalyste et poète. Insistance, 2(14), 101-102. doi: 10.3917/insi.014.0101

Germano, I. M. P., Carneiro, J. S., Pontes, L. M. A., Silva, P. B., \& Gomes, R. C. C. (2018). Eu no facebook: percepções de usuários sobre imagens pessoais partilhadas na rede. Psicologia em Revista, 24(2), 482-505. doi: 10.5752/P.1678-9563.2018v24n2p482-505

Lima, A. F. D., \& Ciampa, A. D. C. (2017). "Sem pedras o arco não existe": o lugar da narrativa no estudo crítico da identidade. Psicologia \& Sociedade, 29(e171330), 1-10. doi: 10.1590/1807-0310/2017v29171330

Lima, A. F. D., Germano, I. M. P., Sabóia, I. B., \& Freire, J. C. (Orgs.). (2018). Sujeito e subjetividades contemporâneas: estudos do Programa de Pós-Graduação em Psicologia da UFC. Fortaleza: Edições UFC.

Linhares, C. (2018). A mancha, o quadro, o signo: sobre a imagem pictórica em Benjamin e Lacan/The stain, the painting, the sign: about the pictorial image in Benjamin and Lacan. Cadernos Benjaminianos, 14(2), 107-125. doi: 10.17851/2179-8478.14.2.107-125

Méllo, R.P. (2018). Cuidar? De quem? De quê? A ética que nos conduz. Curitiba: Appris.

Menezes, J. A., Colaço, V. D. F. R., \& Adrião, K. G. (2018). Implicações políticas na pesquisa-intervenção com jovens. Revista de Psicologia, 9(1), 8-17. Recuperado de http://www.periodicos.ufc.br/psicologiaufc/ article/view/20638

Miranda, L. L., Oliveira, P. S. N., Souza Filho, J. A. D., \& Sousa, S. K. R. B. (2018). A Relação universidade-escola na formação de professores: reflexões de uma pesquisa-intervenção. Psicologia: Ciência e Profissão, 38(2), 301-315. doi: 10.1590/1982-3703005172017

Moura Jr., J. F., \& Sarriera, J. C. (2017). As relações entre pobreza e bem-estar: uma revisão sistemática. Estudos Interdisciplinares em Psicologia, 8(2), 100-125. doi: 10.5433/2236-6407.2017v8n2p100

Oliveira, E. N. P. D., Moita, D. S., \& Aquino, C. A. B. D. (2016). O empreendedor na era do trabalho precário: relações entre empreendedorismo e precarização laboral. Revista Psicologia Política, 16(36), 207-226. Recuperado de http://pepsic.bvsalud. org/scielo.php?script=sci_arttext\&pid=S1519-549X20160002000 06\&lng=pt\&tIng=pt

Pascual, J. G., Ximenes, V. M., Moura Jr., J. F., \& Melo, L. A. (Orgs.). (2012). Experiências entrelaçadas no mestrado: formação para docência. Curitiba: CRV.

Ramos, M. Y. (2018). Internacionalização da pós-graduação no Brasil: lógica e mecanismos. Educação e Pesquisa, 44(e161579), 1-22. doi: 10.1590/s1517-9702201706161579

Santos, B.S. (2014). Do pós-moderno ao pós-colonial. E para além de um e outro. Conferência de abertura do VIII Congresso Luso-Brasileiro de Ciências Sociais realizado de 16 a 18 de setembro de 2014 em Coimbra. Recuperado de https://www.ebah.com.br\&gt

Santos, W. S., Holanda, L. C., Meneses, G. O., Luengo, M., \& GomezFraguela, J. A. (2019). Antisocial behauiour: a unidimensional or multidimensional construct? Avances en Psicología Latinoamericana, 37(1), 13-27. doi: 10.12804/revistas.urosario.edu.co/apl/a.5105 
Severiano, M. D. F. V. (2017). Aceleração social e cultura digital: novas formas de dominação. Comunicações, 24(2), 83-101. doi: 10.15600/2238-121X/comunicacoes.v24n2p83-101

Silva, C. M., Freire, J. C., \& Acselrad, M. (2019). A ética nas famílias contemporâneas. MATRIZes, 13(1), 255-278. doi: 10.11606/issn.19828160.v13i1p255-278

Silva, H. G. S., Bomfim, Z. A. C., \& Costa, J. L. O. (2019). Paisagem, fotografia e mapas afetivos: um diálogo entre a geografia cultural e a psicologia ambiental. Geosaberes, 10(21), 1-22. doi: 10.26895/ geosaberes.v10i21.696

Ximenes, V. M., Nepomuceno, B. B., Cidade, E. C., \& Moura Jr., J. F. (Orgs.). (2016). Implicações psicossociais da pobreza. Fortaleza: Expressão Gráfica e Editora.

Ximenes, V. M., Sarriera, J. C., Bomfim, Z. A. C., \& Alfaro, J. (Orgs.). (2016). Psicologia comunitária no mundo atual: desafios, limites e fazeres. Fortaleza: Expressão Gráfica e Editora.

João Paulo Pereira Barros, Doutor em Educação pela Universidade Federal do Ceará - UFC, é Professor Adjunto da Universidade Federal do Ceará - UFC. Endereço para correspondência: Av. da Universidade, 2762, Bairro Benfica, Fortaleza, Ceará. Telefone: (85) 99734-3012. Email: joaopaulobarros@ufc.br

Cássio Adriano Braz de Aquino, Doutor e Pós-Doutor em Psicologia Social pela Universidad Complutense de Madrid - UCM, é Professor Titular da Universidade Federal do Ceará - UFC. Email: brazdeaquino@gmail.com

Luciana Lobo Miranda, Doutora em Psicologia pela PUC-RIO, Pós-Doutora em Psicologia Social pela City University of New York - CUNY, é Professora Associada da

Universidade Federal do Ceará - UFC. Email: lobo.lu@uol.com.br

Ricardo Pimentel Mello, Doutor e Pós-Doutor em Psicologia Social pela Pontifícia Universidade Católica de São Paulo - PUC-SP, é Professor Titular da Universidade Federal do Ceará - UFC. Email: ricardopmello@gmail.com

Verônica Morais Ximenes, Doutora em Psicologia pela Universidad de Barcelona, Espanha Pós-doutora pela Universidade Federal do Rio Grande do Sul - UFRGS, é Professora Titular da Universidade Federal do Ceará - UFC. Email: vemorais@yahoo.com.br

Veriana de Fátima Rodrigues Colaço, Doutora em Educação pela Universidade Federal do Rio Grande do Sul - UFRGS, Pós-doutora em Psicologia pela Universidade de Barcelona, é Professora Titular da Universidade Federal do Ceará - UFC. Email:verianac@gmail.com

Aluísio Ferreira de Lima, Doutor e Pós-Doutor em Psicologia Social pela Pontifícia Universidade Católica de São Paulo - PUC-SP, é Professor Associado da Universidade Federal do Ceará - UFC. Email: aluisiolima@hotmail.com

Zulmira Aurea Cruz Bomfim, Doutora em Psicologia Social pela Pontifícia Universidade Católica de São Paulo - PUC-SP, Pós doutora em Psicologia Ambiental pela Universidade da Coruña -UDC, Espanha, é Professora Titular da Universidade Federal do Ceará - UFC. Email: zulaurea@gmail.com

Walberto Silva dos Santos, Doutor em Psicologia Social pela Universidade Federal da Paraíba - UFPB, é Professor Associado da Universidade Federal do Ceará- UFC. Email: walbertosantos@gmail.com

Karla Patrícia Holanda Martins, Doutora em Teoria Psicanalítica pela Universidade Federal do Rio de Janeiro - UFRJ, Pós-doutora em Psicologia Clínica pela Universidade de São Paulo - USP, é Professora Associada da Universidade Federal do Ceará - UFC. Email:kphm@uol.com.br 